\title{
Profiling-Based Mobile Advertisement as a Marketing Strategy for GPS- Based Online Traffic Map
}

\author{
${ }^{\#}$ Faculty of Information Technology, Swiss German University \\ EduTown BSD City, Tangerang, Indonesia 15339 \\ ${ }^{1}$ sonny.pradipta@student.sgu.ac.id \\ ${ }^{2}$ sri.endarnotodstudent.sgu.ac.id \\ ${ }^{3}$ james. purnama@sgu.ac.id \\ *Center for Information and Communication Technology (PTIK), \\ Agency for the Assessment and Application of Technology (BPPT) \\ Jalan M.H. Thamrin No. 8, Jakarta, Indonesia 10340 \\ ${ }^{4}$ asnugrohodieee.org \\ $\$$ Jatis Mobile \\ Jalan Mampang Prapatan Raya No.3, Jakarta, Indonesia 12790 \\ ${ }^{5}$ franciscus.pawitraejatis.com
}

Sonny Pradipta ${ }^{\# 1}$, Sri Krisna Endarnoto ${ }^{\# 2}$, James Purnama ${ }^{\# 3}$, Anto S. Nugroho ${ }^{* 4}$, Franciscus C. Pawitra ${ }^{\$ 5}$

\begin{abstract}
The emergence of mobile computing is inevitably followed by mobile advertising: advertising that target mobile device, such as feature phones, smartphones and tablets. However, the majority of mobile advertising is still relying on the traditional approach: to send an advertisement to as many people as possible, in hope that some of them will be interested in the advertisement and in turn buying the promoted product or service. The problem with this method is that each people have their own preferences, so that kind of strategy won't get an optimum result. Furthermore, with traditional strategy there is very low chance of people getting the right advertisement in the right place.

This research proposed a new advertisement system that combined a location based service with user profiling system. In this study, the advertisement system is integrated to an online traffic map mobile application, which also has an RSS feed reader feature. The system will learn user interest by using web mining to analyse browsing history that is taken from the RSS reader. When the user tries to generate a route in the map, the system will automatically fetch advertisements that are located along the route, which suited user interest.

With this proposed system, we have successfully created a mobile advertisement that is highly efficient and effective. Moreover, it has gained positive feedback from user by being accurate, beneficial, and non-obtrusive. Better reception of advertisement from user will lead to the increasing rate of advertisement success.
\end{abstract}

Keywords - marketing intelligence, user profiling, web mining, mobile marketing, location-based service

\section{INTRODUCTION}

Mobile computing has been rapidly growing into such level that it is now widely accepted as a new means of communication. This growth sure brings both opportunities and challenge. In the terms of advertisement alone, studies and reports have showed proof that mobile advertisement is rising as the new form of advertisement [1]-[3]. On the other hand, current mobile advertising is still using traditional approach: to send an advertisement to as many people as possible, in hope that some of them will be interested in the advertisement and in turn buying the promoted product or service. This method has neglected the fact that each people have their own preferences, background and goal in using application, so they won't accept just any kind of advertisement [4].

Mobile computing also enables people to get information as they are going mobile, and they expected to get highly relevant ones from the vast information on the Internet. The key success of information provider is delivering this kind of information through user profiling to get content personalisation [5]. However, traditional approach doesn't take this into consideration and as a result people can't get the right advertisement in the right place.

To answer those problems, this research aim to combine location based service with user profiling system in order to create a new advertisement system. To create user profile, the system use web mining technique to extract information from user's browsing history [6]. After that, system will match advertisement with user profile and location and give the bestsuited advertisement for the given condition.

With this new method of advertisement, people can get a non-obtrusive advertisement that understands them, in term of 


\section{International Conference on Electrical Engineering and Informatics}

17-19 July 2011, Bandung, Indonesia

interest and location. Thus it will lead to better reception of advertisement, which in turn leads to the increase of advertisement success.

This paper is divided into four sections. In Section II, the methodology that is used in this research is discussed. In Section III, the early experiment and the result will be explained. Finally, this paper will be concluded in Section IV.

\section{Methodology}

As shown in Fig.1, this advertisement system can basically be broken down into three parts: input system, user profiling engine, and output system. Input system deals with browsing history data collection. User profiling engine utilize Support Vector machine (SVM) algorithm to perform web content mining to the collected data and extract user interest from it. Output system responsible for matching advertisement with user profile and location, and also display it in the map.

In this study, the advertisement system is integrated to an Android online traffic map mobile application, which utilizing Google Map API. This mobile application also has an RSS feed reader feature. For this prototype, it only included RSS feed from www.detik.com. When user uses the RSS reader, the browsing history will be collected and send to server to be analysed by user profiling engine. User interest will then be extracted and save to database. Once user utilizes the map feature to generate route, the system will automatically fetch advertisements that are: i) located along the route, and ii) suited user interest.

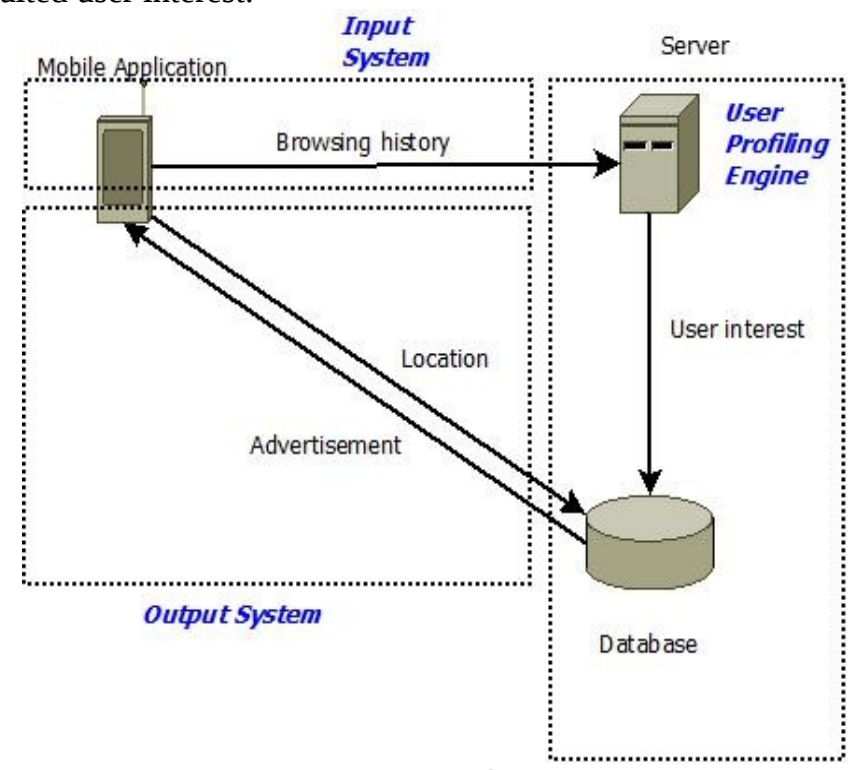

Fig.1 System Architecture

\section{A. Input System}

This system is used to collect browsing history data from the user and do it with user concern. Once user select to read an article in the RSS reader, the article's address link will be saved in an xml file. Periodically, this xml file will be sent to the server to be analysed.



\section{B. User Profiling Engine}

A traditional way to create a user profile is by prompt user to fill their profile manually. Recently, some studies has tried to create a user profiling model by means of information extraction [7]-[9]. However, information such as user interest can't be extracted from the website using those methods. On the other hand, research also shown that we can utilize web content mining to get topic-specific concepts that is implied from the web [10].

Fig. 2 shows the flowchart of this user profiling engine. In this study, a data mining tool called Weka is used to perform web content mining technique [11][12]. The system will read the browsing history data from the xml file, and fetch the content of the websites. Afterwards, this data will be converted into ARFF file, an ASCII file that holds a list of instances which are sharing a set of attributes [12]. This file is exclusively used as Weka own format.

The next step is to classify the news into several predetermined categories. In the experiment, 4 categories are used: sport, dining, entertainment, and health. To do the classification, SVM method with Sequential Minimal Optimization (SMO) is employed because SVM has shown to produce good result given enough training data [13],[14]. Using this technique, user interest can be inferred from the articles that are selected. User interest in this sense is defined as two of the top category that is most frequently selected by user. This system takes into account that user interest can change overtime, by keep on updating and analysing user browsing history. The result will then be saved into the database, which the design is shown in Fig.3. In this phase of research, the prototype system still use Weka manually, as a system to automatically analyse new input data and save them to database is not implemented yet. 


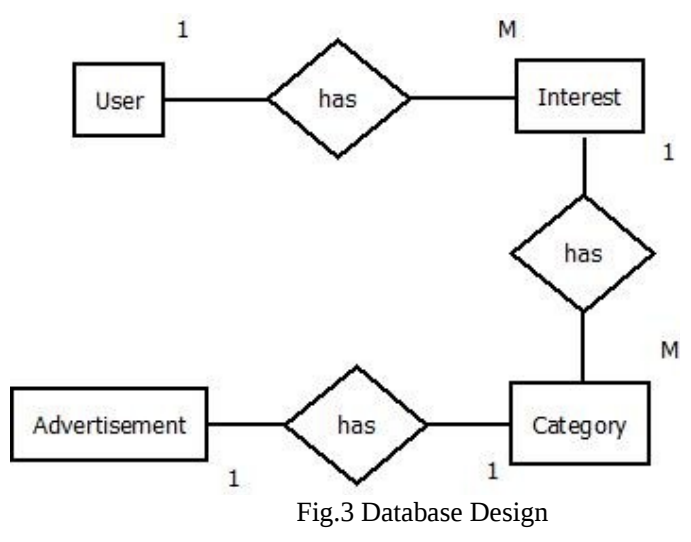

C. Output System

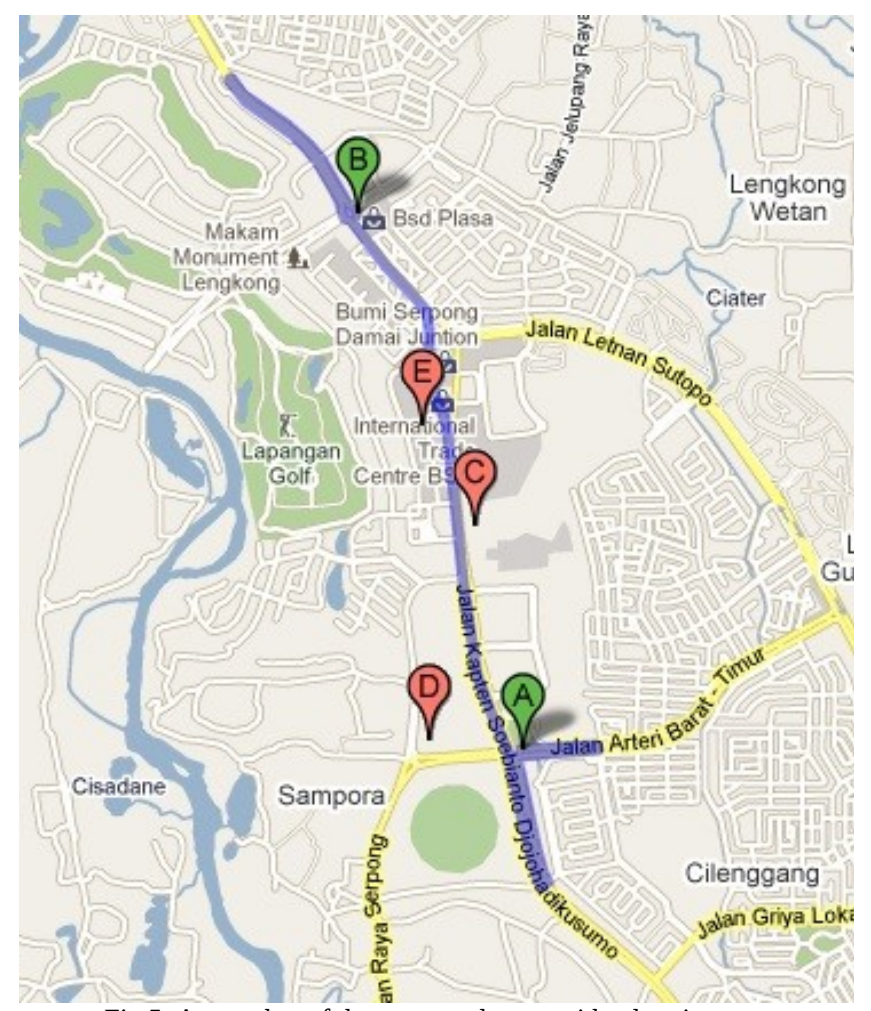

Fig.5 A snapshot of the generated route with advertisement

When user use map application to generate a route from one location to another, it will calculate user position using GPS. This data will be sent to the server, along with a request to find advertisements that fulfil two conditions: matching user interest and matching user area, which is within $1 \mathrm{~km}$ radius of user-generated route. The server will then send back advertisement to the mobile application to be displayed on the map.

Fig. 5 shows the result of the output system. In this example, user try to find a route from point A to point B. This route is generated using Google Map API, and consist of an array of location point (latitude, longitude). The system will then perform a radius check for every point. If there is any advertisement that is located inside the area (less or equal 1 $\mathrm{km}$ from every point), that advertisement data will be sent to the mobile application and displayed in the map, in this case as point $C, D$, and $E$. If user click any advertisement point (C, $\mathrm{D}$, or E) a message box will appear and inform them of what kind of advertisement that shop is having.

\section{RESULT}

The efficiency of the system is analysed by surveying a group of 20 people in BSD City, Tangerang, Indonesia for a period of 3 days. The mobile application is installed in their various GPS-enabled mobile devices (smartphones and tablets). They used the mobile application for 3 days and at the end they were asked to answer a survey about the accuracy of i) user profiling and ii) mobile advertisement. The response for the questions is simply 'yes' or 'no'. For user profiling, they were showed the result of the user interest analysis and were asked if it matched their interest. 95\% of them agree with the analysis and said that the result matched their interest. However, the other $5 \%$ gave a vague answer, so it was categorized as 'no'.

The users were further asked about the result of mobile advertisement, whether it is matched their interest and location. Again, 95\% of them agree that the system provide them with accurate result, which is matched their interest and location. However, all users that previously answered 'yes' in the first question also gave positive result in the second question. That means that the system holds $100 \%$ accuracy if user interest was determined correctly. We didn't test the accuracy of user location, as it is not a control variable. GPS accuracy is determined by Google Map API and sensitivity of GPS in each mobile device, so it will be vary because the users used many types of mobile device. Besides the accuracy, all users also expressed satisfaction of this advertisement system and found it beneficial and non-obtrusive.

This early experiment only consists of 20 users and only classifying user interest into four categories. Furthermore, the test is done in such limited time. If the amount of user and categories, as well as the testing period is increased, the efficiency of the system could be proven to be higher than the previous result.

\section{CONCLUSIONS}

This research designed and implemented an advertisement system that can recognize both user profile and location. User profile is taken from user's browsing history, which will be classified into several categories by using web mining technique to extract user interest. When user tries to generate a route in the mobile application, the system will send advertisement that matched both user interest and the route that is seek.

Early experiments have proved that this new system is highly efficient and effective. It also gained approval of user, by being beneficial and non-obtrusive. With this new method of advertisement, people can get a non-obtrusive 


\section{International Conference on Electrical Engineering and Informatics}

17-19 July 2011, Bandung, Indonesia

advertisement that understands them, in term of interest and location. Thus it will lead to better reception of advertisement, which in turn leads to the increase of advertisement success. However, there is much work to be done for the overall improvement of this proposed system, particularly in the automation of user profiling engine, as it is done semimanually now. Moreover, a wider variety of user interest categories and a deeper experiment which cover a greater number of participants and testing period to get a better accuracy of this system.

\section{ACKNOWLEDGMENT}

This research wouldn't be completed without the help of Michael Scof and Reggio Hartono from Swiss German University, and also Rinto Priambodo and Rindang Septyan from Jatis Mobile.

\section{REFERENCES}

[1] IAB \& PricewaterhouseCooper, "IAB Internet Advertising Revenue Report 2010,” 2011. [Online]. Available: http://www.iab.net/ AdRevenueReport

[2] Morgan Stanley, “The Mobile Internet Report Setup,” 2009. [Online.] Available: http://www.morganstanley.com/institutional/techresearch

[3] Morgan Stanley. “Internet Trends,” 2010. [Online.] Available: http://www.morganstanley.com/institutional/techresearch

[4] S. Schiaffino and A. Amandi, "Intelligent user profiling," Artificial Intelligence: an international perspective, vol. 1, pp 193-216, 2009.
[5] B. Rousseau, P. Browne, P. Malone, \& M. OFoghlú, "User profiling for content personalisation in information retrieva,l" in Proc. 19th Annual Conference of ACM Symposium on Applied Computing, 2004.

[6] M. Grčar, D. Mladenič, \& M. Grobelnik, "User profiling for interestfocused browsing history," in Proc. 8th international multi-conference on Information Society, 2005, pp 182-185.

[7] H. Alani, S. Kim, D.E. Millard, M.J. Weal, W. Hall, P.H. Lewis, \& N.R. Shadbolt, "Automatic ontology-based knowledge extraction from web documents,” IEEE Intelligent Systems, vol. 18, pp 14-21, 2003.

[8] M.J. Pazzani \& D. Billsus, "Learning and revising user profiles: the identification of interesting websites," Machine Learning, vol. 27, pp 313-331, 1997.

[9] K. Yu, G. Guan, \& M. Zhou, "Resume information extraction with cascaded hybrid model,” in Proc. 43rd Annual Meeting on Association for Computational Linguistics (ACL'05), 2005, pp 499-506.

[10] B. Liu, C.W. Chin, \& H.T Ng, "Mining topic-specific concepts and definitions on the web," in Proceedings of the 12th international conference on World Wide Web, 2003, pp 251-260.

[11] S.R. Garner, "Weka: The waikato environment for knowledge analysis," in Proc. New Zealand computer science research students conference, 1995, pp 57-64.

[12] M. Hall, E. Frank, G. Holmes, B. Pfahringer, P. Reutemann, \& I.H Witten, "The WEKA data mining software: an update," ACM SIGKDD Explorations Newsletter, vol. 11, pp 10-18, 2009.

[13] C.H. Chan, A. Sun, \& E.P. Lim, "Automated Online News Classification with Personalization," in Proc. 4th International Conference of Asian Digital Library, 2001.

[14] J. Platt, "Sequential minimal optimization: A fast algorithm for training support vector machines," Advances in Kernel Methods-Support Vector Learning, vol. 208, pp 98-112, 1999. 